\title{
Composite supercapacitor electrodes made of activated carbon/PEDOT:PSS and activated carbon/doped PEDOT
}

\author{
T S SONIA，P A MINI，R NANDHINI，KALLURI SUJITH，BALAKRISHNAN AVINASH, \\ S V NAIR* and K R V SUBRAMANIAN* \\ Amrita Centre for Nanosciences and Molecular Medicine, Ponekkara P. O., Kochi 682 041, Kerala, India
}

MS received 21 December 2011; revised 5 July 2012

\begin{abstract}
In this paper, we report on the high electrical storage capacity of composite electrodes made from nanoscale activated carbon combined with either poly(3,4-ethylenedioxythiophene):poly(styrenesulfonate) (PEDOT:PSS) or PEDOT doped with multiple dopants such as ammonium persulfate (APS) and dimethyl sulfoxide (DMSO). The composites were fabricated by electropolymerization of the conducting polymers (PEDOT:PSS, doped PEDOT) onto the nanoscale activated carbon backbone, wherein the nanoscale activated carbon was produced by ball-milling followed by chemical and thermal treatments. Activated carbon/PEDOT:PSS yielded capacitance values of $640 \mathrm{~F} \mathrm{~g} \mathrm{~g}^{-1}$ and $26 \mathrm{mF} \mathrm{cm}{ }^{-2}$, while activated carbon/doped PEDOT yielded capacitances of $1183 \mathrm{~F} \mathrm{~g}^{-1}$ and $42 \mathrm{mF} \mathrm{cm} \mathrm{cm}^{-2}$ at $10 \mathrm{mV} \mathrm{s}$. This is more than five times the storage capacity previously reported for activated carbon-PEDOT composites. Further, use of multiple dopants in PEDOT improved the storage performance of the composite electrode well over that of PEDOT:PSS. The composite electrodes were characterized for their electrochemical behaviour, structural and morphological details and electronic conductivity and showed promise as high-performance energy storage systems.
\end{abstract}

Keywords. Carbon materials; composite materials; electrodeposition; energy storage and conversion; thin films; conducting polymers.

\section{Introduction}

Poly(3,4-ethylenedioxythiophene) (PEDOT) is one of the best known $p$-conjugated conducting polymers. Because of their excellent properties (Ryu et al 2004; Snooke et al 2010) (high conductivity, high stability, transparency in the oxidized state and relatively low bandgap), PEDOT and its derivatives have developed into one of the most important materials from both fundamental and practical perspectives and are now utilized in several applications, such as LEDs, organic solar cells and electrochromic devices Wang and Wong (2006). During the initial stages of development, PEDOT that was synthesized using the conventional oxidative chemical/electrochemical method was insoluble in water. However, the solubility problem was overcome using a water-soluble polyelectrolyte, poly(styrenesulfonic acid) (PSS) (Yang et al 2008). PSS was also found to help in keeping the PEDOT segments dispersed in water. PSS also acts as the charge-balancing dopant for PEDOT, thus providing the desired electrical properties. When processed with organic solvents such as glycerol and dimethyl sulfoxide (DMSO), conductivity of the films is enhanced by two orders of magnitude (Antje et al 2009). This phenomenon was initially attributed to the screening effects of polar solvents on Coulomb interaction between positively charged PEDOT

*Author for correspondence (krvsubramanian@aims.amrita.edu and shantinair@aims.amrita.edu) grain and negatively charged PSS dopant (Alexandre 2007) or to a solvent-induced conformational change in the PEDOT chain, which increases the interchain interaction. Recent investigations indicate that this conductivity enhancement is due to morphological changes in the film structure and a phase-segregation process. Thus, the dispersion of PEDOT segments is attributed to the water solubility of PSS and Coulombic interactions between PEDOT and PSS, which promote hydrophilicity through dipole interactions.

Poly(3,4-ethylenedioxythiophene):poly(styrenesulfonate) (PEDOT:PSS) consists of a conducting polythiophene derivative that is electrostatically bound to a PSS polyanion. Since this polymer has high electrical conductivity, processibility, high stability, flexibility and good transparency, it has found a wide variety of applications including polymeric anodes for organic photovoltaics, light-emitting diodes (Pingree et al 2008), flexible electrodes, supercapacitors (Huang et al 2006) and electrochromic devices.

Research in the area of supercapacitors has been done on composites for a wide number of materials especially with carbon-related materials. The major carbon materials that are looked upon are carbon nanotubes (CNTs), graphene and activated carbon. Specific capacitances of up to as high as $300 \mathrm{~F} / \mathrm{g}$ in an aqueous electrolyte has been achieved for activated carbon electrodes (Zheng and Jow 1995). Activated carbon fibres modified by ruthenium chloride showed up to $180 \mathrm{~F} / \mathrm{g}$ offering good characteristics for use in supercapacitors (Wang and $\mathrm{Hu}$ 2005). Activated carbon fibres with high 
surface area and highly mesoporous structure have been prepared from polyacrylonitrile with $\mathrm{NaOH}$ activation. These yielded up to $371 \mathrm{~F} / \mathrm{g}$ making them suitable for high performance EDLCs (Xu et al 2008). Very high surface area activated carbon $\left(3000 \mathrm{~m}^{2} / \mathrm{g}\right)$ yielded up to $300 \mathrm{~F} / \mathrm{g}$ for use in supercapacitors (Obreja 2008). PEDOT/carbon nanotubes (CNTs) composite supplied lower capacitance values of $100 \mathrm{~F} \mathrm{~g}^{-1}$ (Frackowiak et al 2006) with a perfect stability during cycling. Additional advantage of the PEDOT/CNTs composite was a possibility of operating in acidic, alkaline and organic electrolytic solutions. Composite electrodes of PEDOT and CNTs were prepared (Lota et al 2004) by (a) direct polymerization on ultrasonically dispersed CNTs (130 $\mathrm{F} \mathrm{g}^{-1}$ ) and (b) electrochemical deposition of polymer onto the CNTs $\left(150 \mathrm{~F} \mathrm{~g}^{-1}\right)$. Also, a negatively charged CNT suspension and PEDOT was co-deposited (Peng et al 2006) and gave capacitances of atleast $0.5 \mathrm{~F} \mathrm{~cm}^{-2}$.

An electrode made of PEDOT-carbon composite exhibited high specific capacitance due to the semiregular, macroporous nature of the electrode film. The composite electrode was prepared with multi-walled carbon nanotubes by chemical or electrochemical polymerization of PEDOT on nanotubes and the capacitance is reported as $60-160 \mathrm{~F} / \mathrm{g}$ (Kelly et al 2009; Sharma and Zhai 2009). An important work was reported on enhancement of double-layer capacitance behaviour and electrical conductivity in layered PEDOT based nanocomposite (Murugan et al 2005). Also there are works done on PEDOT and related materials such as PEDOT-graphene composite (Xu et al 2009), PEDOTCNTs composites $\left(150 \mathrm{~F} \mathrm{~g}^{-1}\right.$ ) (Lota et al 2004) and activated carbon-PEDOT composites $\left(158 \mathrm{~F} \mathrm{~g}^{-1}\right.$ ) (Selvakumar and Bhat 2008). These advances give a way for many other combinations of carbon-conducting polymer composites for energy storage and other applications.

In our paper, we developed composite electrodes with high electrical storage capacity made from nanoscale activated carbon combined with either poly $(3,4-$ ethylenedioxythiophene):poly(styrenesulfonate) (PEDOT: PSS) or PEDOT doped with multiple dopants such as ammonium persulfate (APS) and dimethyl sulfoxide (DMSO). The composites were fabricated by electropolymerization of the conducting polymers (PEDOT:PSS, doped PEDOT) onto the nanoscale activated carbon backbone. The composite carbon-doped PEDOT electrodes showed high specific capacitance, which is more than five times the storage capacity previously reported for activated carbon-PEDOT composites. Further, the use of multiple dopants in PEDOT improved storage performance of the composite electrode well over that of PEDOT:PSS.

\section{Materials and methods}

\subsection{Preparation of activated carbon}

Graphite powder is taken and subjected to rotary ball milling for $11 \mathrm{~h}$ with the usage of balls made up of tungsten carbide, hardness of balls being 14 on Moh scale and speed of rotation of the mill being $300 \mathrm{rpm} .1 \mathrm{~g}$ of the ball milled graphite is mixed with $20 \mathrm{ml}$ of $\mathrm{HNO}_{3}$ and ultrasonicated for $3 \mathrm{~h}$ at $60{ }^{\circ} \mathrm{C}$. A given volume of the supernatant is taken and further mixed with equal parts of $\mathrm{H}_{2} \mathrm{SO}_{4}$ and ultrasonicated for $3 \mathrm{~h}$ at $60{ }^{\circ} \mathrm{C}$. The resulting solution containing carbon particles is washed sufficiently with de-ionized water to remove traces of acid and air dried.

\subsection{Electropolymerization of PEDOT:PSS}

PEDOT monomer and PSS was purchased from M/s SigmaAldrich. PEDOT was not distilled before use. Electropolymerization was carried out in a 3-electrode set up with the working electrode as titanium, saturated calomel as reference electrode and platinum as counter electrode with the voltage being $1.8 \mathrm{~V}$ and polymerization times being 25,50 and $100 \mathrm{~s}$. The electrolyte consisted of 0.05 M PEDOT and 0.005 g PSS in $10 \mathrm{ml}$ of distilled water.

\subsection{Electropolymerization of doped PEDOT}

PEDOT was multiple doped with 0.01 M (ammonium persulfate (APS), a common oxidant for polymerization) and 5 microlitres of dimethyl sulfoxide (DMSO). APS and DMSO were added to $0.05 \mathrm{M}$ PEDOT solution in $10 \mathrm{ml}$ water and further electropolymerized at $1.8 \mathrm{~V}$ for different time intervals $(15,25,50$ and $100 \mathrm{~s})$.

\subsection{Preparation of activated carbon/PEDOT:PSS and activated carbon/doped PEDOT composites}

Composite studies were carried out on activated carbon (ball milled size, $150 \mathrm{~nm}$ chemically treated) by depositing PEDOT:PSS and doped PEDOT onto it and then comparing the results. $2 \mathrm{mg}$ of activated carbon in $10 \mathrm{ml}$ of isopropanol was electrophoretically deposited on titanium (cathode), anode being platinum rod. Conditions were $20 \mathrm{~V}$ for $20 \mathrm{~min}$. Subsequently, the layer was annealed in air at $100{ }^{\circ} \mathrm{C}$ for $3 \mathrm{~h}$. Both polymers were electrodeposited on activated carbon at $1.8 \mathrm{~V}$ for $100 \mathrm{~s}$. Cyclic voltammetry $(\mathrm{CV})$ studies on the composite electrodes were carried out in $0.1 \mathrm{M}$ $\mathrm{LiClO}_{4}$ in acetonitrile at various scan rates.

\section{Results and discussion}

The activated carbon, as described in $\S 2.1$, was analysed for its particle size using SEM and the average particle size was $150 \mathrm{~nm}$. In our reported studies (Nandhini et al 2012), this activated carbon was made as an electrode by electrophoretic deposition and tested for its specific capacitance using CV studies in $0.1 \mathrm{M} \mathrm{KOH}$. The activated carbon yielded a specific capacitance of $413 \mathrm{~F} \mathrm{~g}^{-1}$ at $100 \mathrm{mV} \mathrm{s}^{-1}$. The activated 
carbon was further used as a conducting and high surface area backbone for electrodepositing PEDOT:PSS and doped PEDOT polymers.

Initial studies were carried out on electropolymerized PEDOT:PSS on titanium substrates as outlined in $\S 2.2$ which shows that optimum polymerization time is $50 \mathrm{~s}$. This yields maximum specific and area capacitances of $197 \mathrm{~F} \mathrm{~g}^{-1}$ and $24.6 \mathrm{mF} \mathrm{cm}^{-2}$. The morphology shows a porous globular structure. The Raman spectra of PEDOT:PSS has major peaks at $1440 \mathrm{~cm}^{-1}$ which corresponds to the $C_{\alpha}=C_{\beta}$ stretching of the structure and at $1596 \mathrm{~cm}^{-1}$ corresponding to the asymmetric stretching of $C_{\alpha}=C_{\beta}$ bond.

Figure 1 shows SEM image of the activated carbonPEDOT:PSS composite which was synthesized as per $\S 2.4$. The activated carbon/PEDOT:PSS composite yielded capacitance values of $640 \mathrm{~F} \mathrm{~g}^{-1}$ and $26 \mathrm{mF} \mathrm{cm}{ }^{-2}$ at $10 \mathrm{mV} \mathrm{s}^{-1}$. The electrochemical CV curves are shown in figure 2. The high value of specific capacitance is because the activated carbon provides a high surface area, porous and conductive backbone for electropolymerization and this kind of morphology maximizes the faradaic interactions with the electrolyte.

Surface current profile measurements of the PEDOT:PSS thin film/doped PEDOT thin film and the activated carbonPEDOT:PSS composite/activated carbon-doped PEDOT composite were carried out in a scanning electrochemical microscope (SECM, make: Sensolytics) with the help of $10 \mu \mathrm{m}$ wider platinum microelectrode, $\mathrm{Ag} / \mathrm{AgCl}$ reference electrode and $0 \cdot 1 \mathrm{M} \mathrm{LiClO}_{4}$ in acetonitrile as the electrolyte. The activated carbon-PEDOT:PSS/activated carbon-doped PEDOT composite yielded higher values of current reinforcing the role played by the nanoscale activated carbon in providing a conductive backbone (figures $3(\mathrm{a}, \mathrm{b})$ and $4(\mathrm{a}, \mathrm{b})$ ). The current scale on each graph is on the right top and a darker shade on the 3-D plot shows higher value of current. PEDOT was doped with various dopants like DMSO, sorbitol

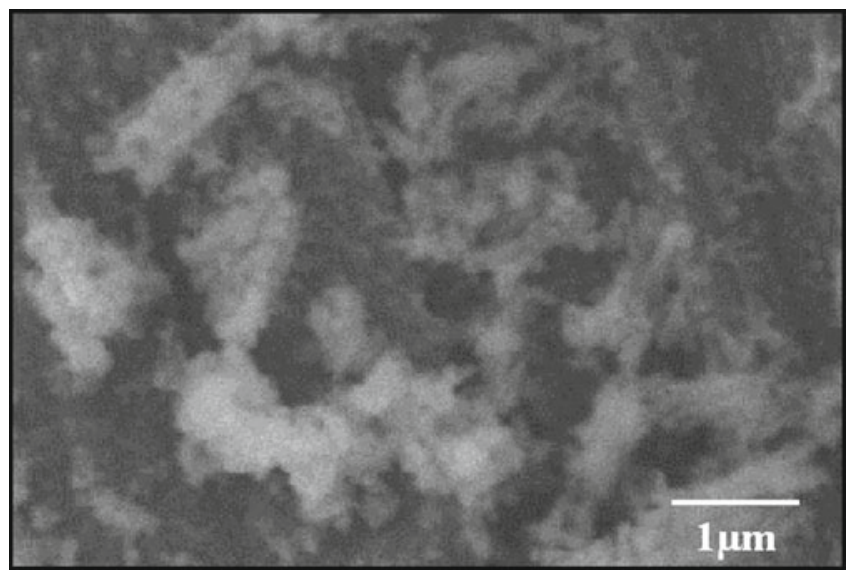

Figure 1. High magnification SEM image of activated carbonPEDOT:PSS composite showing polymer film deposited over underlying carbon backbone.

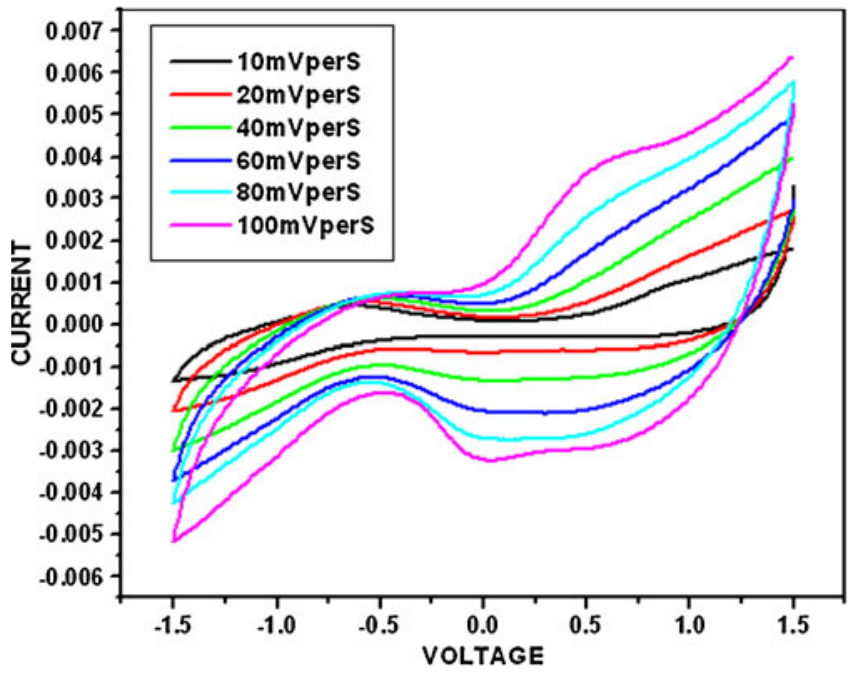

Figure 2. CV curves of activated carbon-PEDOT:PSS composite at various scan rates in $0.1 \mathrm{M} \mathrm{LiClO}_{4}$ in acetonitrile. Calomel electrode is used as reference. Scan rates are varied from $10 \mathrm{mV} \mathrm{s}^{-1}$ to $100 \mathrm{mV} \mathrm{s}^{-1}$.

and APS and the electrochemical performance of the electrodeposited thin films were individually measured. Based on the CV studies, 0.01 M APS $+5 \mu$ l of DMSO was chosen as the doping media for PEDOT. Films were polymerized as per $\S 2.3$ and polymerization for $25 \mathrm{~s}$ gave the optimum electrochemical data $\left(230 \mathrm{~F} \mathrm{~g}^{-1}\right.$ at $\left.10 \mathrm{mV} \mathrm{s}^{-1}\right)$.

Composite electrodes of the activated carbon-doped PEDOT were synthesized as per $\S 2.4$. The activated carbon/doped PEDOT composite yielded capacitance values of $1183 \mathrm{~F} \mathrm{~g}^{-1}$ and $42 \mathrm{mF} \mathrm{cm}^{-2}$ at $10 \mathrm{mV} \mathrm{s}^{-1}$. The electrochemical CV curves are shown in figure 5. The morphology of the activated carbon-doped PEDOT composite is shown in figure 6. Doping PEDOT with multiple dopants can affect the self-discharge characteristics. Self-discharge, to a large extent, is dependant on the internal resistance/impedance of the active layer in the electrode. Thus, on account of higher electrode/active mass conductivities (figures 3 and 4, which are also representative of lower internal resistance), we expect self-discharge to be within acceptable limits even with multiple doping.

FTIR data for both activated carbon/PEDOT:PSS and activated carbon/doped PEDOT composite is shown in figure 7. The appearance of additional peaks is shown for the composites when compared to FTIR of carbon alone. The nanoscale activated carbon-doped PEDOT composite electrodes showed high specific capacitance, which was more than five times the storage capacity previously reported for activated carbon-PEDOT composites. The large capacitance values that we have achieved is due to high surface area provided by the nanoscale activated carbon together with its conductivity and this provides an optimum backbone for polymer deposition. Thus, surface area for faradaic interactions of the porous conducting polymer with the electrolyte 


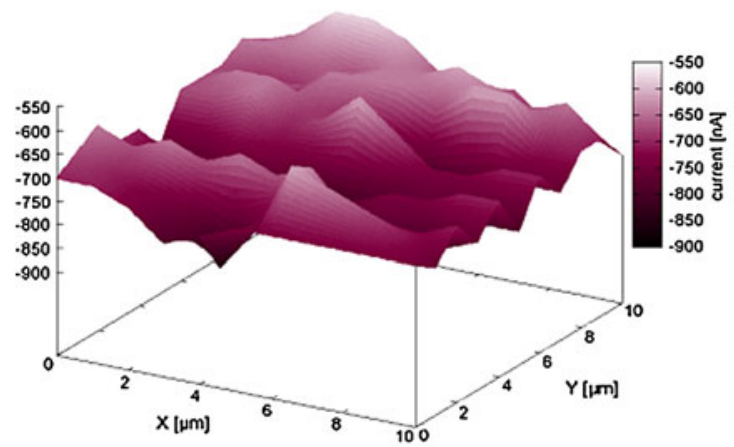

a) SECM OF PEDOT:PSS

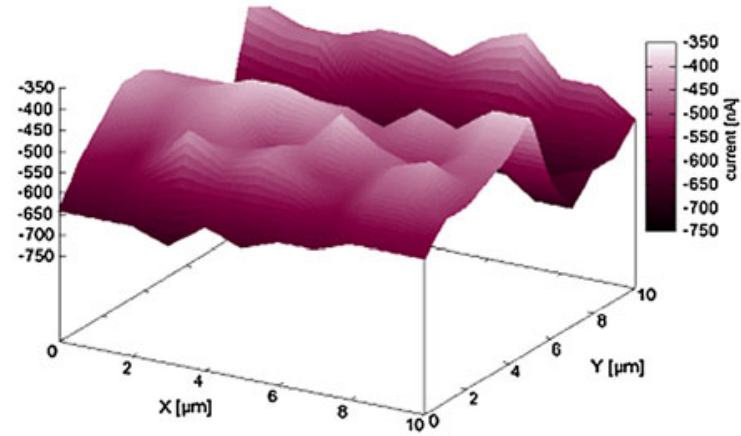

b) SECM OF CARBON + PEDOT:PSS

Figure 3. SECM 3-D current plot for PEDOT:PSS and activated carbon-PEDOT:PSS composite. Current bar in nA is shown on top right of plot. Darker shade indicates higher values of current.

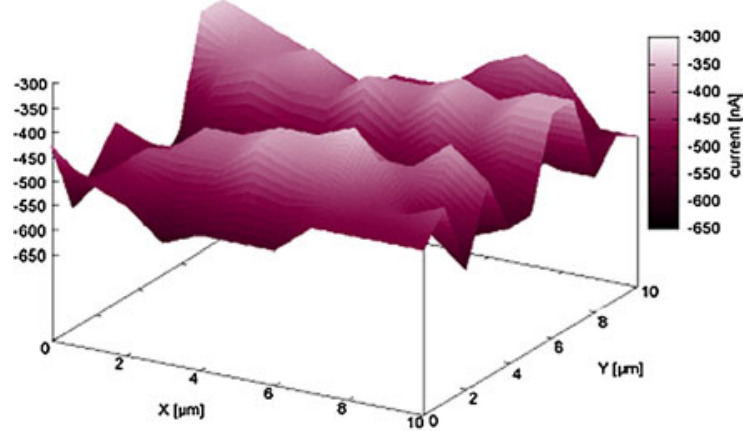

a) SECM OF DOPED PEDOT

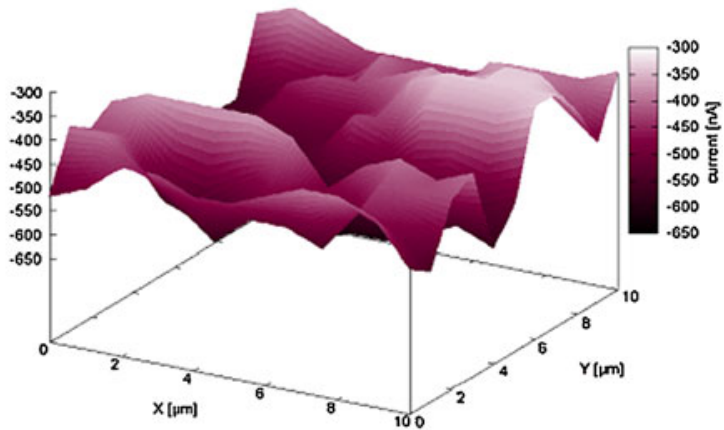

b) SECM OF CARBON+ DOPED PEDOT

Figure 4. SECM 3-D current plot for doped PEDOT and activated carbon-doped PEDOT composite. Current bar in $\mathrm{nA}$ is shown on top right of plot. Darker shade indicates higher values of current.

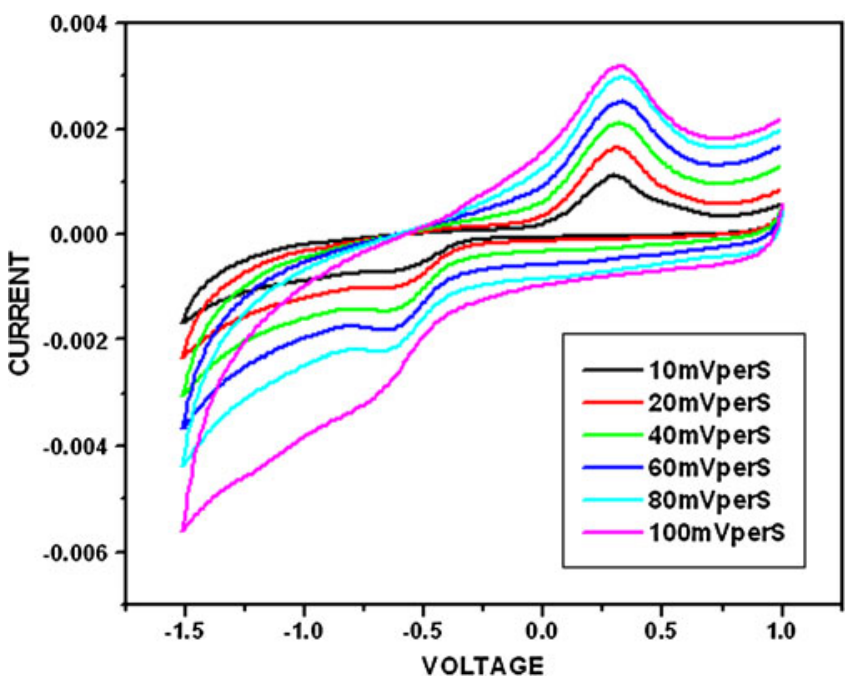

Figure 5. CV curves of activated carbon-doped PEDOT composite at various scan rates in $0 \cdot 1 \mathrm{M} \mathrm{LiClO}_{4}$ in acetonitrile. Calomel electrode is used as reference. Scan rates are varied from $10 \mathrm{mV} \mathrm{s}^{-1}$ to $100 \mathrm{mV} \mathrm{s}^{-1}$.

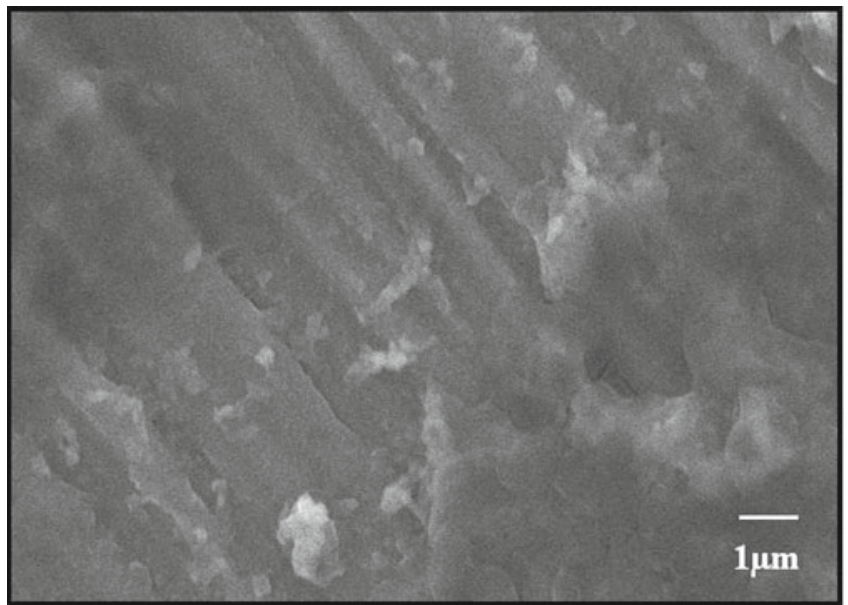

Figure 6. SEM image of activated carbon-doped PEDOT composite showing polymer film deposited over underlying carbon backbone. 


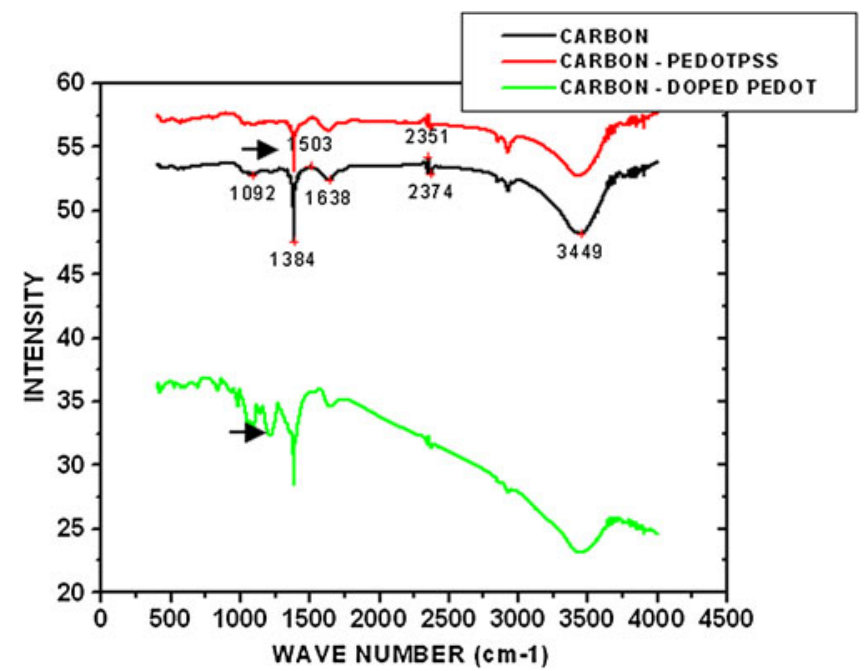

Figure 7. FTIR data for carbon alone, activated carbon-PEDOT: PSS composite and activated carbon-doped PEDOT composite. Upon composite formation, additional peaks appear in FTIR spectrum (shown by arrows).

is also enhanced. Further, the use of multiple dopants in PEDOT improved the storage performance of the composite electrode well over that of PEDOT:PSS.

\section{Conclusions}

In conclusion, we were able to successfully synthesize composite supercapacitor electrodes with high electrical storage capacity made from nanoscale activated carbon combined with either PEDOT:PSS or PEDOT doped with multiple dopants such as APS and DMSO. The nanoscale activated carbon-doped PEDOT composite electrodes showed high specific capacitance of $1183 \mathrm{~F} \mathrm{~g}^{-1}$, which is more than five times the storage capacity previously reported for activated carbon-PEDOT composites. Further, the use of multiple dopants in PEDOT improved the storage performance of the composite electrode well over that of PEDOT:PSS $\left(640 \mathrm{~F} \mathrm{~g}^{-1}\right)$.

\section{Acknowledgements}

The authors acknowledge the Department of Science and Technology, Government of India and the Ministry of New and Renewable Energy, Government of India, for supporting this work. The authors are also indebted to the Amrita Institute of Medical Sciences for the infrastructure support extended to this programme.

\section{References}

Alexandre M N 2007 Technical Report, Eindhoven University, The Netherlands

Antje S, Abdiaziz A F, Arun A and Amr S H 2009 J. Phys. Chem. B113 9352

Frackowiak E, Khomenko V, Jurewicz K, Lota K and B'eguin F 2006 J. Power Sources 153413

Huang L, Lin H, Wen T C and Gopalan A 2006 Electrochim. Acta 521058

Kelly T L, Yano K and Wolf M O 2009 Applied Mater. Interf. ACS 12536

Lota K, Khomenko V and Frackowiak E 2004 J. Phys. Chem. Solids 65295

Murugan A V, Kasi Viswanathan A, Campet G, Gopinath C S and Vijayamohanan K 2005 Appl. Phys. Lett. 871

Nandhini R, Mini P A, Avinash B, Nair S V and Subramanian K R V 2012 Mater. Lett. 87165

Obreja V V N 2008 Physica E40 2596

Peng C, Snook G A, Fray D J, Shaffer M S P and Chen G Z 2006 Chem. Commun. 4629

Pingree L S C, Macleod B A and Ginger D S 2008 J. Phys. Chem. C112 7922

Ryu K S, Lee Y G, Hong Y S, Park Y J, Wu X and Kim K M 2004 Electrochim. Acta $\mathbf{5 0} 843$

Selvakumar M and Bhat D K 2008 J. Appl. Polym. Sci. 1072165

Sharma R K and Zhai L 2009 Electrochim. Acta $\mathbf{5 4} 7148$

Snooke G A, Kao P and Best A S 2010 J. Power Sources 133031

Wang X J and Wong K Y 2006 Thin Solid Films 5151573

Wang C C and Hu C C 2005 Carbon 431926

Xu Y, Wang Y, Liang J, Huang Y, Ma Y and Wan X 2009 Nano Res. 2343

Xu B, Wu F, Chen R, Cao G, Chen S, Zhou Z and Yang Y 2008 Electrochem. Commun. 10795

Yang Y, Jiang Y, Xu J and Yu J 2008 Thin Solid Films 5161191

Zheng J P and Jow T R 1995 J. Electrochem. Soc. 142 L6 